\title{
Tarification à l'activité et financement des médicaments onéreux en France : que peut-on en apprendre? Comparaison de deux démarches d'encadrement de ces médicaments
}

\author{
Jean-François Bussières, Jennifer Corny et Olivier Bourdon
}

\section{INTRODUCTION}

T es dépenses en santé ont crû à un rythme supérieur à l'inflation Ldans la plupart des pays occidentaux au cours des deux dernières décennies ${ }^{1}$. Cette hausse soutenue est notamment liée au vieillissement de la population, à l'utilisation croissante des services de santé, dont le recours aux professionnels et aux médicaments, et à la hausse des dépenses liées à la rémunération des professionnels. Parallèlement à cette hausse, plusieurs États ont limité la hausse des prix des médicaments à l'indice des prix à la consommation sans pour autant contrôler les prix à l'entrée sur le marché des nouveaux médicaments ${ }^{2}$.

En 2012 en France, la part des dépenses en médicaments représentait 15,2\% des dépenses en santé contre 13,4\% des dépenses en santé au Canada en $2014^{3}$.

Dans ce contexte, la France s'est tournée en 2007 vers le financement des établissements basé sur la tarification à l'activité (T2A) plutôt que sur l'historique des dépenses. En 2014, la Direction générale de l'offre de soins a publié son rapport au Parlement, relatif à la réforme du modèle de financement des établissements de santé . Ce rapport, précise que l'objectif de la T2A est de " réduire les inégalités de santé [...] et de faire progresser à la fois la démocratie sanitaire et l'efficience de notre système de santé ». Cependant, des limites ont été mises en évidence, comme l'augmentation incessante de l'activité et une tarification ne dépendant pas de la qualité des soins.

Or et coll. ont évalué l'activité, la productivité et la qualité des soins des hôpitaux avant et après la T2 $\mathrm{A}^{5}$. Les auteurs notent " une tendance à la hausse de la productivité [...] dans le secteur public depuis 2004 tandis que dans le secteur privé lucratif, on observe une modification de l'activité (case-mix) plus qu'une augmentation marquée de la production et de la productivité ". Selon les auteurs, la T2A a créé des « incitations directes pour augmenter le nombre des séjours et réduire les coûts par séjour auxquels les établissements peuvent répondre de manière variable $»^{5}$. Une revue systématique de Palmer et coll. publiée en 2014 a également permis de recenser et d'analyser 65 études menées dans 10 pays afin d'évaluer les retombées du financement à l'activité sur de nombreux indicateurs ${ }^{6}$. Les auteurs concluent que le passage à la T2A est associé à une hausse substantielle du taux de réadmissions, tandis que les effets sur les autres indicateurs varient selon les études. Cependant, aucun indicateur spécifique à l'utilisation des médicaments n'a été utilisé. Les chercheurs proposent d'envisager ce modèle de tarification avec prudence, compte tenu des répercussions et des difficultés inhérentes à cette transition.

Au Québec, l'idée de tarification à l'activité fait l'objet de discussions et de recommandations depuis au moins deux décennies, notamment par l'entremise du rapport Bédard sur la budgétisation et la performance financière des centres hospitaliers publié en $2002^{7}$. Plus récemment, en 2012, le ministère de la Santé et des Services sociaux a mis en place un groupe d'experts chargé d'étudier le financement à l'activité ${ }^{8}$. En février 2014, ce groupe d'experts a publié son rapport, qui propose un modèle de financement axé sur le patient ${ }^{9}$. Dans le cadre de la $41^{\mathrm{e}}$ législature, le projet de loi 10, sanctionné le 9 février 2015, prévoit notamment " un nouveau mode d'allocation des ressources financières pour le financement du réseau. Les budgets pour les établissements seront désormais versés en fonction des programmes-services (ex. : santé publique, santé physique, déficience intellectuelle, dépendances, santé mentale) et non plus sur une base historique ${ }^{10}$. 
Il existe très peu de documents décrivant les répercussions et les enjeux inhérents à la budgétisation des établissements de santé au Canada sur le budget en médicaments et les activités d'un département de pharmacie. Bussières et coll. se sont intéressés à la possibilité de recourir à la tarification à l'activité pour le financement des médicaments onéreux en établissements de santé en $2010^{11}$. À l'époque, les auteurs étaient d'avis que le modèle français devrait profiter d'une étude descriptive de faisabilité sur des données issues de centres universitaires. Dans ce contexte, nous avons développé dans notre centre une démarche d'encadrement de l'utilisation de médicaments dits émergents, y compris des médicaments non approuvés au Canada, des médicaments approuvés utilisés dans des indications non approuvées et des médicaments très onéreux.

Nous avons donc souhaité décrire le mécanisme de financement des médicaments onéreux en France et le comparer au système de médicaments émergents mis en place dans notre centre.

\section{DESCRIPTION DU PROGRAMME}

Il s'agit d'une étude descriptive. L'objectif principal visait à décrire le mécanisme d'encadrement du financement et de l'utilisation des médicaments en France. L'objectif secondaire était de comparer le contenu de la liste des médicaments hors groupe homogène de séjour (GHS) au contenu de la liste des médicaments émergents utilisés dans notre centre canadien, et de nous interroger sur l'applicabilité d'un tel mode de financement des médicaments au Québec. Un groupe homogène de séjour correspond à un diagnostic auquel est associé un tarif " tout-inclus » de remboursement fixé d'avance. Les médicaments hors groupe homogène de séjour peuvent être remboursés lorsque leur utilisation respecte des critères supplémentaires à ceux du séjour lui-même.

L'étude porte sur l'ensemble des médicaments hors GHS ou émergents. Ont toutefois été exclus les médicaments radiopharmaceutiques et les dérivés du sang, qui ne sont généralement pas gérés par les pharmaciens au Canada, afin de renforcer la comparabilité des deux listes.

\section{Mécanisme d'encadrement du financement et de l'utilisation des médicaments en France}

À partir d'une revue documentaire succincte, nous avons revu les mécanismes d'encadrement du financement et de l'utilisation des médicaments en France et proposé un schéma synthèse afin de réfléchir aux possibilités et aux enjeux de cette approche au Canada.

\section{Médicaments hors groupes homogènes de séjour (GHS) - France}

La liste des médicaments hors GHS en cours le 19 janvier 2015 a été extraite des référentiels de bon usage du médicament sur le portail web de l'Agence nationale de sécurité du médicament
(ANSM). Les données extraites étaient les suivantes : dénomination commune internationale, spécialité pharmaceutique, type d'autorisation d'accès au marché (français/européen), mode d'encadrement de l'utilisation (c.-à-d. indications dans le cadre de l'autorisation de mise sur le marché, protocoles thérapeutiques temporaires [PTT], situations avec données insuffisantes répertoriées [hors groupe] dans les référentiels de bon usage ou situations non acceptables [SNA]) et classes thérapeutiques. Les indications potentielles relatives à chacune des spécialités pharmaceutiques ayant des PTT, des SNA ou des indications hors groupe ont été extraites du portail web de l'ANSM.

\section{Médicaments émergents — Québec}

En 2014, une liste de médicaments classés comme émergents a été mise en place dans notre centre hospitalier universitaire pour permettre l'encadrement de l'utilisation de nouveaux médicaments, notamment parce qu'ils sont couteux, que les données probantes à leur sujet sont limitées et que leur utilisation est nouvelle et croissante. Cette liste, créée à partir d'une revue rétrospective d'utilisation des médicaments correspondant à ces critères, est mise à jour régulièrement selon les demandes des prescripteurs. Cette liste a été extraite le 19 janvier 2015. Les données extraites étaient les suivantes : dénomination commune internationale, spécialité pharmaceutique, type d'autorisation d'accès au marché par spécialité (avis de conformité de Santé Canada, Programme d'accès spécial), mode d'encadrement de l'utilisation, classes thérapeutiques, indications autorisées dans notre centre.

Ensuite, une comparaison de ces listes a eu lieu selon les données extraites de chacune des listes.

\section{ÉVALUATION DU PROGRAMME}

\section{Mécanisme d'encadrement du financement et de l'utilisation des médicaments en France}

En ce qui concerne le mécanisme d'encadrement du financement et de l'utilisation des médicaments en France, un établissement de santé se voit rembourser pour chaque pathologie selon un prix fixe prédéterminé par groupe homogène de séjour. À cela viennent s'ajouter des montants pour certains services particuliers, en fonction des missions d'enseignement et de recherche (p. ex. urgences, greffe) et pour les cliniques externes et les consultations spécialisées.

Lorsqu'il faut recourir à des médicaments et à des dispositifs médicaux onéreux (listes établies par le ministère de la Santé), un financement distinct est accordé en sus de la tarification basée sur les GHS ${ }^{12}$. Ces médicaments onéreux, dits hors GHS, seront financés à $100 \%$ du prix d'acquisition, mais l'assurance maladie pourrait réduire ce taux à $95 \%$, voire à $70 \%$. Afin d'être remboursé à $100 \%$, l'établissement hospitalier doit avoir signé un contrat de bon usage (CBU) et en respecter les modalités, notamment la déclaration honnête des indications et des 
utilisations. Le prescripteur doit justifier par écrit dans le dossier médical du patient l'indication reconnue dans l'autorisation de mise sur le marché (groupe 1) ou dans un protocole thérapeutique temporaire (protocole d'utilisation dans une indication non approuvée mais autorisée et encadrée par les autorités, groupe 2). On ne devrait pas observer d'utilisation de ces médicaments dans des situations non acceptables (groupe 3). En revanche, il se peut que l'on doive recourir à des médicaments pour des indications non approuvées pour manque de données (hors groupe), notamment dans les centres hospitaliers universitaires, mais cette utilisation doit être basée sur des données publiées ou être dument justifiées. Certaines de ces indications sont reconnues au niveau national (Référentiels de bon usage de l'ANSM). Les autres dépendent généralement des pathologies rencontrées dans les hôpitaux. Ce respect des indications fait partie du contrat de bon usage, signé entre les établissements et l'Agence régionale de santé $^{11}$. L'informatisation de ce système permet aux chefs de départements de pharmacie de consulter ces justificatifs dans une base de données locale.

La figure 1 présente une vue d'ensemble des mécanismes d'encadrement du financement et de l'utilisation des médicaments en France, dans le cadre de la tarification à l'activité.

\section{Comparaison du contenu de la liste des médicaments hors GHS au contenu de la liste des médicaments émergents utilisés dans notre centre}

Au 19 janvier 2015, la liste française des médicaments hors GHS comportait 143 spécialités pharmaceutiques. Toutefois, 50 d'entre elles ont été exclues de notre comparaison (médicaments radiopharmaceutiques ou médicaments dérivés du sang). Au total, 38 spécialités pharmaceutiques pouvaient être utilisées dans une indication en vertu d'un PTT, mais 38 autres ne pouvaient l'être en vertu d'un statut de situation non acceptable. Soixantedix-huit (78) des 143 spécialités pharmaceutiques détenaient un avis de conformité au Canada.

À cette même date, la liste des médicaments émergents dans notre centre comportait 106 spécialités pharmaceutiques (57 dénominations communes internationales, DCI), dont 17 étaient présentées dans la liste de médicaments hors GHS.

Aucune des indications présentes dans les PTT ou dans les SNA n'a été retenue sur la liste des médicaments émergents de notre centre. Toutefois, une indication correspondait à une situation avec insuffisance de données (azacitidine pour le traitement de la leucémie aigüe myéloblastique lors de rechute en pédiatrie).

Le tableau 1 présente le profil des médicaments inscrits dans la liste hors GHS en France ou dans la liste des médicaments émergents de notre centre en 2015.

\section{RÉPERCUSSIONS SUR LA PRATIQUE}

Cette étude descriptive permet de mettre en parallèle deux processus distincts d'encadrement de l'utilisation des médicaments en France et au Canada et leurs conséquences sur leur financement, qui convergent vers des objectifs similaires et une utilisation optimale des médicaments. Le nombre de dénominations communes internationales inscrites à la liste des médicaments émergents de notre centre est gérable $(n=57)$ et compatible avec le nombre de DCI figurant sur la liste des médicaments hors GHS établie en France $(n=97)$ pour une patientèle plus large, bien que seules 17 DCI étaient communes aux deux listes. Cette différence peut notamment s'expliquer par le fait que les immunoglobulines et les autres médicaments dérivés du sang sont inscrits à la liste française, mais ne sont généralement pas gérés par les pharmaciens au Canada et ne font donc pas partie de notre liste de médicaments émergents. En outre, il existe un décalage entre l'approbation des médicaments par l'Agence européenne du médicament et par Santé Canada, de sorte que certains médicaments ne sont pas forcément approuvés, à un moment déterminé, par les deux entités juridiques.

Cette démarche de comparaison de la liste de médicaments hors GHS en France avec notre liste locale s'inscrit dans un processus d'amélioration de la qualité des soins et de réflexion sur les répercussions de l'implantation éventuelle de la tarification à l'activité au Canada. Elle permet notamment de cibler les médicaments dont il est possible d'envisager l'utilisation en dehors de l'avis de conformité.

L'approche proposée au sein de notre centre vise à encadrer non seulement l'utilisation de médicaments émergents, comme le fait la liste française, mais aussi l'incertitude liée aux preuves disponibles et aux couts le plus souvent importants de ces thérapies. Si l'approche française est fondée sur une confirmation $\mathrm{du}$ respect de l'indication établie par un tiers externe sans forcément faire appel à d'autres intervenants et au patient, l'approche développée dans notre centre comporte les différences suivantes : il s'agit d'un processus local, qui suppose la participation directe d'au moins quatre cliniciens (c.-à-d. médecin traitant, chef médical de service, pharmacien traitant, chef de département de pharmacie) invités à justifier, sur un formulaire type joint au dossier du patient, l'indication visée, les marqueurs de résultats et les délais compatibles avec une réponse thérapeutique, les marqueurs d'innocuité et le plan de traitement. En outre, le formulaire vise à confirmer que le prescripteur a obtenu le consentement libre, manifeste et éclairé du patient/parent et qu'il a discuté des enjeux de remboursement possible en usage ambulatoire. L'annexe 1 (publié au : www.cjhp-online.ca/ index.php/cjhp/issue/view/114/showToc) illustre le formulaire type implanté pour encadrer les médicaments émergents au sein de notre établissement.

Il nous semble primordial que chaque centre universitaire canadien se dote d'un système d'encadrement de l'utilisation de ces médicaments onéreux. La liste française de médicaments hors GHS et la démarche d'encadrement des médicaments émergents de notre centre représentent des exemples de mécanisme pouvant être mis en place. 


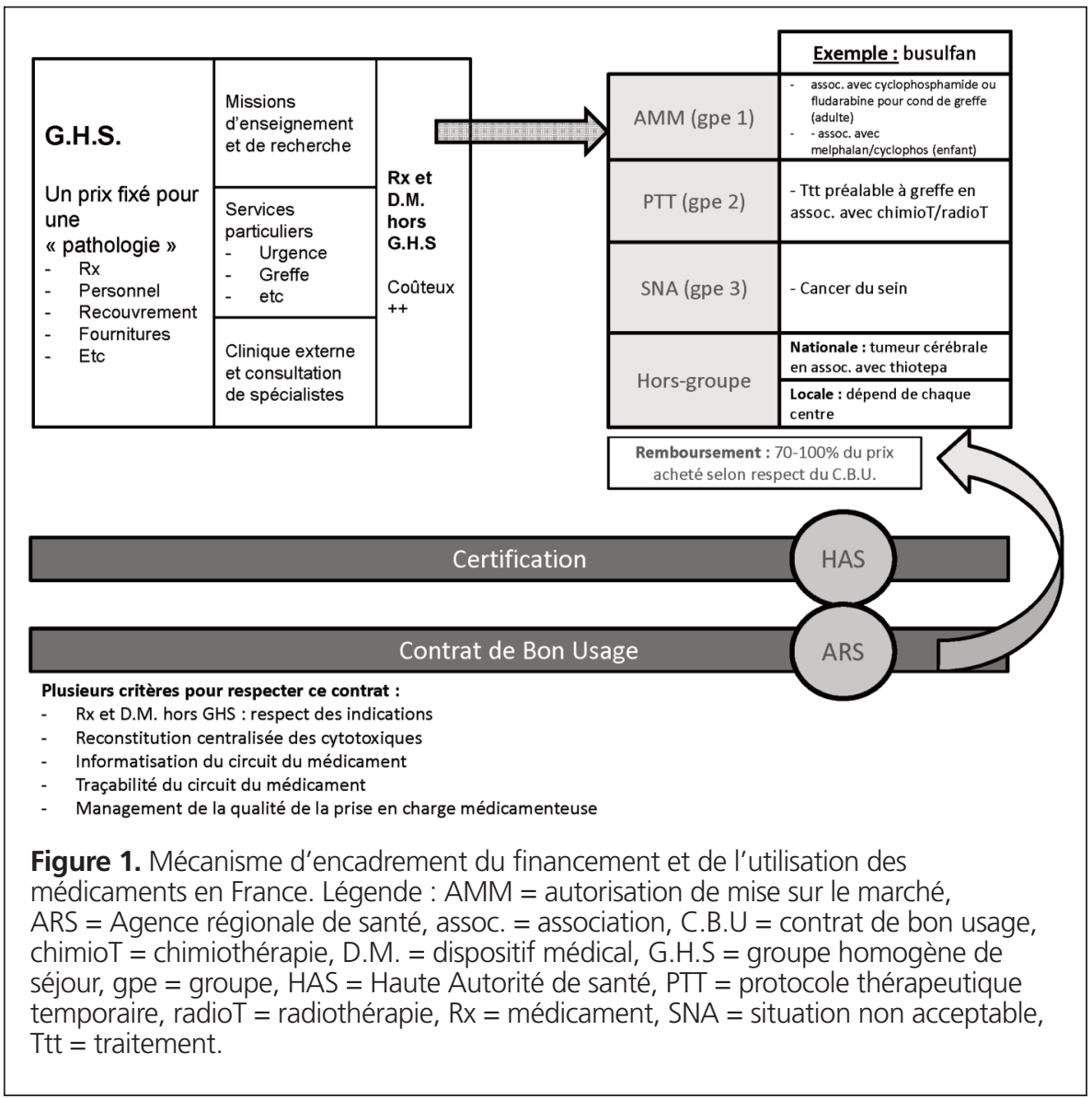

Tableau 1. Profil des médicaments inscrits sur la liste hors GHS en France ou sur la liste de médicaments émergents dans un CHU canadien en 2015

\begin{tabular}{lcc} 
& France & CHU canadien \\
\hline Liste de médicaments & Médicaments hors GHS & Médicaments émergents \\
\hline Spécialités pharmaceutiques & 143 & 106 \\
\hline Dénominations communes & 97 & 57 \\
internationales & & \\
\hline Spécialités par type d'autorisation & & \\
d'accès au marché & 78 & $\mathrm{NA}$ \\
Européenne & 65 & $\mathrm{NA}$ \\
Française & $\mathrm{NA}$ & 90 \\
Santé Canada & $\mathrm{NA}$ & 16 \\
Programme d'accès spécial & & $\mathrm{NA}$ \\
Spécialités par mode d'encadrement & 143 & $\mathrm{NA}$ \\
Autorisation de mise sur le marché & 38 & $\mathrm{NA}$ \\
Protocole thérapeutique temporaire & 38 & $\mathrm{NA}$ \\
Situation non acceptable & $\mathrm{ND}$ & 106 \\
Hors groupe & $\mathrm{NA}$ & \\
Émergent & & 62 \\
Spécialités par classes thérapeutiques & 45 & 0 \\
Anticancéreux & 8 & 1 \\
Rx associés aux anticancéreux & 6 & 0 \\
Antifongiques et antibiotiques & 5 & 6 \\
Anti-TNF alfa & 10 & $\mathrm{NA}$ \\
Déficit enzymatique & 35 & $\mathrm{NA}$ \\
Facteurs de la coagulation & 11 & 4 \\
Immunoglobulines & 10 & 33 \\
Hypertension artérielle pulmonaire & 13 & \\
Autres & & \\
CHU = centre hospitalier universitaire, GHS = groupe homogène de séjour, NA & non applicable, \\
ND = données non disponibles, Rx = médicaments, & NFF tumor necrosis factor (facteur de \\
nécrose tumorale). & & \\
& &
\end{tabular}


Comme le soulignent quelques études et quelques rapports ayant évalué les répercussions de la tarification à l'activitétece changement fondamental du mode de financement des établissements de santé n'est pas sans conséquences sur les résultats relatifs à la santé et à d'autres aspects, si bien que les chefs de départements de pharmacie canadiens devraient assurer une veille électronique de cette littérature, sachant que ce type de financement risque d'être implanté dans plus d'une province. De plus, les chefs de départements de pharmacie devraient se doter d'outils d'analyse permettant d'estimer les couts au cas par cas afin de déterminer à l'avance les écarts dans le financement proposé par le tiers payeur public.

De plus, le changement de mode de financement est susceptible de s'accompagner de mesures administratives visant à expliquer les écarts entre la prescription et les indications acceptées. Par exemple, une circulaire administrative a été mise en place en 2007 par le ministère de la Santé et des Services sociaux afin d'encadrer l'utilisation du bévacizumab ${ }^{13}$. Une telle approche ne peut être utilisée pour des dizaines de médicaments, et il faut envisager un outil intégré au prescripteur électronique et au dossier pharmacologique informatisé pour espérer une méthode de collecte d'information efficiente.

En outre, toute démarche d'encadrement de l'émergence doit se faire dans l'esprit du cadre juridique en vigueur. Au Québec, il revient au chef du département de pharmacie de déterminer le contenu de la liste des médicaments disponibles au sein de l'établissement, en tenant compte des recommandations du comité de pharmacologie. Dans le même esprit, l'encadrement de l'émergence doit profiter de l'expertise du pharmacien, qui a une vue globale de l'ensemble des besoins de tous les patients traités et qui peut proposer justement une utilisation optimale des fonds disponibles.

Cette étude comporte des limites. Il s'agit d'une étude descriptive transversale. De nouveaux médicaments sont commercialisés régulièrement, et le processus d'ajout d'un médicament à la liste hors GHS ou à la liste des médicaments émergents est dynamique. Compte tenu que la liste des médicaments émergents dans notre centre n’a été mise en place qu'à l'automne 2014, une évaluation longitudinale sur une période de 12 mois pourrait donner des résultats plus globaux. En outre, notre centre est un établissement mère-enfant, de sorte que de nombreux médicaments de la liste hors GHS ne comportent pas d'indications pour une pharmacothérapie de mères et d'enfants.

\section{CONCLUSION}

Cette étude descriptive nous a permis de décrire le mécanisme d'encadrement du financement et de l'utilisation des médicaments onéreux en France et a permis de nous interroger sur la comparaison des médicaments concernés avec le système mis en place dans notre centre. Dans notre centre mère-enfant, la conception d'un mécanisme d'encadrement de l'émergence a été largement inspirée de ce modèle français, bien que des modifications importantes aient été apportées. La liste française comprend notamment de nombreux médicaments peu utilisés en pédiatrie, et cette liste a donc été réduite et adaptée à la patientèle de notre centre. De plus, ce mécanisme d'encadrement de l'émergence vise à justifier non seulement l'utilisation de médicaments émergents, comme le fait la liste française, mais aussi l'incertitude liée aux données probantes disponibles et aux couts souvent importants de ces thérapies. Compte tenu des spécificités de chaque établissement, nous pensons qu'il est important de constituer une liste locale de médicaments émergents, en nous inspirant de la liste hors GHS française par exemple, afin de renforcer localement le bon usage de ces médicaments au Canada.

\section{Références}

1. Métadonnées de la base de données sur les dépenses nationales de santé (BDDNS). Ottawa (ON) : Institut canadien d'information sur la santé; 2015. Publié au : www.cihi.ca/CIHI-ext-portal/internet/fr/document/ spending+and+health+workforce/spending/health+spending/nhex metadata. Consulté le 10 août 2015.

2. Rapport annuel 2013. Ottawa (ON) : Conseil d'examen du prix des médicaments brevetés; 2014. Publié au : www.pmprb-cepmb.gc.ca/ view.asp?ccid=938. Consulté le 10 août 2015.

3. Dépenses en médicaments en 2014. Ottawa $(\mathrm{ON})$ : Institut canadien d'information sur la santé. Publié au : www.cihi.ca/web/resource/fr/ nhex_2014_infosheet_fr.pdf. Consulté le 10 août 2015.

4. Rapport 2014 au Parlement relatif à la réforme du modèle de financement des établissements de santé. Paris (France) : Direction générale de l'offre de soins; 2014. Publié au : www.apmnews.com/Documents/rapportauparlementart 41lfss14.pdf. Consulté le 10 août 2015.

5. Or Z, Bonastre J, Journeau F, Nestrigue C. Activité, productivité et qualité des soins des hôpitaux avant et après la T2A [document de travail]. Paris (France) : Institut de recherche et documentation en économie de la santé; 2013. Publié au : www.irdes.fr/EspaceRecherche/DocumentsDeTravail/ DT56SoinsHospitaliersT2A.pdf. Consulté le 10 août 2015.

6. Palmer KS, Agoritsas T, Martin D, Scott T, Mulla SM, Miller A, et al. Activity-based funding of hospitals and its impact on mortality, readmission, discharge destination, severity of illness, and volume of care: a systematic review and meta-analysis. PLoS One. 2014;9(10):e109975.

7. La budgétisation et la performance financière des centres hospitaliers. Rapport du comité sur la réévaluation du mode de budgétisation des centres hospitaliers de soins généraux et spécialisés. Québec (QC) : Direction des communications du Ministère de la Santé et des Services sociaux; 2002. Publié au : http://publications.msss.gouv.qc.ca/acrobat/f/documentation/2001/01-60402.pdf. Consulté le 10 août 2015.

8. Le Groupe d'experts sur le financement à l'activité dans le réseau de la santé et des services sociaux entame ses travaux [communiqué]. Québec (QC) : Ministère de la Santé et des Services sociaux; 2012. Publié au : www.msss. gouv.qc.ca/documentation/salle-de-presse/ficheCommunique. php?id=340. Consulté le 10 août 2015.

9. Pour que l'argent suive le patient : l'implantation du financement à l'activité axé sur les patients dans le secteur de la santé. Québec (QC) : Groupe d'experts pour un financement axé sur les patients; 2014. Publié au : www.groupes. finances.gouv.qc.ca/santefinancementactivite/wp-content/uploads/ 2014/02/Rapport-Financ-axe-patients-19fev14.pdf. Consulté le 8 janvier 2016.

10. Observatoire franco-québécois de la santé et de la solidarité. Réformes en cours au Québec. Québec (QC) : Ministère de la Santé et Services sociaux Québec; 2015. Publié au : www.msss.gouv.qc.ca/ministere/observatoiresss/ index.php?id=126,8299,0,0,1,0. Consulté le 10 août 2015.

11. Bussières JF, Simoncelli M, Bourdon O, Lachaîne J. Doit-on s'inspirer de la tarification à l'activité pour le financement des médicaments onéreux en établissements de santé? Can J Hosp Pharm. 2010;63(3):236-43. 
12. Référentiels de bon usage des médicaments. Saint-Denis (France) : Agence nationale de sécurité du médicament; 2015. Publié au : http://ansm.sante.fr/ Dossiers/Referentiels-de-bon-usage-des-medicaments/Referentiels-de-bonusage-des-medicaments/(offset)/0. Consulté le 10 août 2015.

13. Annexe 1 à la circulaire 2007-026. Règles d'utilisation du bévacizumab (AvastinMC). Québec (QC) : Ministère de la Santé et des Services sociaux; 2007. Publié au : http://msssa4.msss.gouv.qc.ca/fr/document/d26ngest.nsf/ d1ff67a9711c03238525656b00166b21/1af49f94aee43f16852573640061 4c03/\$FILE/Annexe\%201.pdf. Consulté le 10 août 2015.

Jean-François Bussières, B. Pharm., M. Sc., M. B. A., FCSHP, est chef, Département de pharmacie et Unité de recherche en pratique pharmaceutique, Centre hospitalier universitaire Sainte-Justine, et professeur, Faculté de pharmacie, Université de Montréal, Montréal (Québec).

Jennifer Corny est candidate au Pharm. D. et interne en pharmacie, Assistance publique hôpitaux de Paris, Paris, France. Elle est aussi assistante de recherche, Unité de recherche en pratique pharmaceutique, Centre hospitalier universitaire Sainte-Justine, Montréal, QC.

Olivier Bourdon, Pharm. D., M. Sc., Ph. D., est pharmacien-chef à I'Hôpital universitaire mère-enfant Robert-Debré, Assistance publique hôpitaux de Paris, Paris, France.

Intérêts concurrents : Aucun déclaré.

\section{Adresse de correspondance :}

Jean-François Bussières

Département de pharmacie

Centre hospitalier universitaire Sainte-Justine

3175, chemin de la Côte Sainte-Catherine

Montréal QC H3T 1 C5

Courriel : jf.bussieres@ssss.gouv.qc.ca

Financement : Aucun reçu.

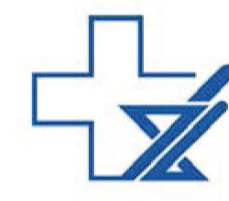

\section{Canadian Society of Hospital Pharmacists Société canadienne des pharmaciens d'hôpitaux}

\section{Membership Year 2016-20I7 (July I, 2016 to June 30, 2017)}

Membership renewal time is here!

We have begun taking renewals for the 2016-2017 year.

Support your profession by remaining part of a vibrant and strongly-networked pharmacy organization. Together, we are the voice of hospital pharmacists, helping to shape the delivery of healthcare in Canada.

Renew today at my.cshp.ca!

Membership Enquiries: Please contact Membership Services Tel.: (6|3) 736-9733 Ext. 222 | Fax: (6I3) 736-5660 | Email: membershipservices@cshp.ca 